\title{
Las calcificaciones simétricas y asimétricas de los ganglios basales pueden reflejar enfermedad mitocondrial
}

\author{
Josef Finsterer ${ }^{1}$ y Concepción Maeztu² \\ ${ }^{1}$ Krankenanstalt Rudolfstiftung, Viena, Austria; ${ }^{2}$ Hospital Clínico Universitario Arrixaca, División de Neuropsicología Clínica, Murcia, España
}

Hemos leído con interés el estudio retrospectivo de Jiménez-Ruiz et al. sobre las causas de la calcificación simétrica de los ganglios basales (CSGB) en una cohorte de pacientes a quienes se realizó tomografía axial computarizada craneal. ${ }^{1}$

El título indica que las calcificaciones eran simétricas, ${ }^{1}$ sin embargo, las Figuras 1, 2 y 3 muestran calcificaciones asimétricas. ¿Cuál fue el grado de asimetría aceptado para incluirlas?

Los autores indican que el periodo de revisión fue de cuatro años, sin embargo, en Métodos se describe que abarcó de 2012 a 2016 (cinco años). ¿Cuál fue el tiempo real de estudio?

Los autores mencionan que la CSGB puede encontrarse en $1 \%$ de las tomografías axiales computarizadas craneales. ${ }^{1}$ Dado que siete pacientes tenían CSGB, esto implica que debieron realizarse aproximadamente 700 tomografías durante el periodo de estudio. ¿Esa fue la cifra correcta o los autores encontraron otro porcentaje de CSGB, como previamente se menciona??

De los siete pacientes, uno tenía hipoparatiroidismo y el otro pseudohipoparatiroidismo. Como estas dos enfermedades pueden tener rasgos fenotípicos de síndrome MELAS, ${ }^{3}$ era esencial que en los pacientes 2 y 3 se investigara enfermedad mitocondrial. ¿Estos dos pacientes tenían otros rasgos fenotípicos que sugirieran enfermedad mitocondrial?

La Tabla 3 indica que la CSGB puede ocurrir en enfermedad mitocondrial como el MERRF, ${ }^{1}$ sin embargo, la CSGB es infrecuente en el MERRF. ${ }^{4}$

Una deficiencia del estudio es que los casos de MELAS no fueron confirmados genéticamente, aun cuando es conocido que por su heterogeneidad ${ }^{5}$ es esencial clarificar la base genética de este síndrome.

\section{Bibliografía}

1. Jiménez-Ruiz A, Cárdenas-Sáenz O, Ruiz-Sandoval JL. Symmetrical and bilateral basal ganglia calcification. Case series and literature review. Gac Med Mex. 2018;154:258-262.

2. Rumboldt Z, Castillo M, Huang B, Huang B, eds. Brain imaging with MRI and CT: an image pattern approach. EE.UU.: Cambridge University Press; 2012.

3. Morten KJ, Cooper JM, Brown GK, Lake BD, Pike D, Poulton J. A new point mutation associated with mitochondrial encephalomyopathy. Hum Mol Genet. 1993;2:2081-2087.

4. Finsterer J, Zarrouk-Mahjoub S, Shoffner JM. MERRF Classification: implications for diagnosis and clinical trials. Pediatr Neurol. 2018;80:8-23.

5. DiMauro S, Hirano M. MELAS. En: Adam MP, Ardinger HH, Pagon RA, Wallace SE, et al., eds. GeneReviews [Internet]. Seattle, WA: University of Washington; 1993-2018. Disponible en: http://www.ncbi.nlm.nih.gov/ books/NBK1233.
Gac Med Mex. 2018;154:501-501

Disponible en PubMed www.gacetamedicademexico.com 